\title{
Light-induced boron-oxygen defect generation in compensated $p$-type Czochralski silicon
}

\author{
D. Macdonald, ${ }^{1, a)}$ F. Rougieux, ${ }^{1}$ A. Cuevas, ${ }^{1}$ B. Lim, ${ }^{2}$ J. Schmidt, ${ }^{2}$ M. Di Sabatino, ${ }^{3}$ and \\ L. J. Geerligs ${ }^{4}$ \\ ${ }_{1}^{1}$ Department of Engineering, College of Engineering and Computer Science, The Australian National \\ University, Canberra ACT 0200, Australia \\ ${ }^{2}$ Institut für Solarenergieforschung Hameln (ISFH), Am Ohrberg 1, D-31860 Emmerthal, Germany \\ ${ }^{3}$ SINTEF Materials and Chemistry, A. Getz v. 2B, 7465 Trondheim, Norway \\ ${ }^{4}$ ECN Solar Energy, P.O. Box 1, NL-1755 ZG Petten, The Netherlands
}

(Received 11 February 2009; accepted 19 March 2009; published online 1 May 2009)

\begin{abstract}
The concentration of boron-oxygen defects generated in compensated $p$-type Czochralski silicon has been measured via carrier lifetime measurements taken before and after activating the defect with illumination. The rate of formation of these defects was also measured. Both the concentration and the rate were found to depend on the net doping rather than the total boron concentration. These results imply that the additional compensated boron exists in a form that is not able to bond with the oxygen dimers, thus prohibiting the formation of the defect. This could be explained by the presence of boron-phosphorus complexes, as proposed in previous work. Evidence for reduced carrier mobilities in compensated silicon is also presented, which has implications for photoconductance-based carrier lifetime measurements and solar cell performance. (C) 2009 American Institute of Physics. [DOI: 10.1063/1.3121208]
\end{abstract}

\section{INTRODUCTION}

Recombination centers caused by boron-oxygen defects are well known in crystalline silicon solar cells. ${ }^{1-3}$ They are thought to consist of a substitutional boron $\left(\mathrm{B}_{s}\right)$ atom complexed with an interstitial oxygen dimer $\left(\mathrm{O}_{2 i}\right)$. ${ }^{4,5}$ Naturally, they are most prevalent in material that is both oxygen rich and boron doped, for example, $p$-type Czochralski silicon, and to a lesser degree, $p$-type multicrystalline silicon. ${ }^{6,7}$ The concentration of the $\mathrm{B}_{s}-\mathrm{O}_{2 i}$ defects has been found to be approximately linearly related to the boron concentration and approximately quadratically related to the interstitial oxygen content. ${ }^{8}$ The $\mathrm{B}_{s}-\mathrm{O}_{2 i}$ complexes are formed under illumination (or by other means of carrier injection) and can be returned to their passive state of isolated $\mathrm{B}_{s}$ and $\mathrm{O}_{2 i}$ by annealing at temperatures around $200{ }^{\circ} \mathrm{C}$. ${ }^{9}$ Further illumination at typical cell operating temperatures then results in reactivation of the defect. Recent work showed that the $\mathrm{B}_{s}-\mathrm{O}_{2 i}$ defects can be converted to a permanently passive state by annealing under illumination. ${ }^{10}$

With the advent of new types of low-cost solar-grade silicon, many of which are compensated, ${ }^{11-15}$ it is interesting to consider the extent of $\mathrm{B}_{s}-\mathrm{O}_{2 i}$ defect creation in compensated silicon. As pointed out recently by Kopecek et al. ${ }^{16}$ and Dubois et al., ${ }^{17}$ at first glance, one might expect greater concentrations of the defect to occur in compensated material compared to noncompensated material of the same net doping because it contains additional boron. Note that in compensated $p$-type silicon, the net doping $p_{0}$ is given by $p_{0}$ $=N_{A}-N_{D}$, where $N_{A}$ and $N_{D}$ are the total ionized acceptor and donor concentrations, respectively. These additional

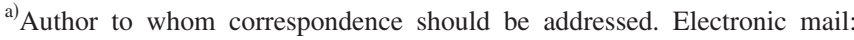
daniel.macdonald@anu.edu.au.
}

$\mathrm{B}_{s}-\mathrm{O}_{2 i}$ defects would lead to reduced carrier lifetimes in solar cells made on such material, in addition to the impact of reduced carrier mobilities expected in compensated material.

However, some recent results based on the measurement of solar cell open-circuit voltages have suggested that the formation of $\mathrm{B}_{s}-\mathrm{O}_{2 i}$ defects is no greater in compensated solar-grade silicon than in comparable noncompensated material. ${ }^{18,16}$ This implies that the additional compensated boron is not able to take part in the defect reaction. Interestingly, Krühler et al. ${ }^{19}$ previously also observed that the dominant recombination process in solar cells made from compensated $p$-type $\mathrm{Cz}$-Si appeared to be proportional to the net doping rather than the boron concentration, leading them to propose the presence of B-P pairs. Kopecek et al. noted that these pairs could neatly explain their own observations. ${ }^{16}$ Such B-P pairs have indeed been observed in single-crystal silicon via low temperature Fourier transform infrared spectroscopy (FTIR), both in material that was compensated in the melt during crystal growth ${ }^{20,21}$ and also after implantation and annealing of compensating dopants. ${ }^{22,23}$

In this work we have used photoconductance-based carrier lifetime measurements to quantify the extent of formation of $\mathrm{B}_{s}-\mathrm{O}_{2 i}$ defects in strongly compensated $\mathrm{Cz}$ silicon that is otherwise very pure. Via a direct comparison of the defect concentration in compensated and noncompensated materials we were able to verify that the $\mathrm{B}_{s}-\mathrm{O}_{2 i}$ defect concentration does indeed depend on the net doping $p_{0}$ rather than the boron concentration $N_{A}$. These conclusions are corroborated by measurements of the defect generation rate, which was also found to depend on $p_{0}$ rather than $N_{A}$. These observations can be explained by the presence of B-P complexes, possibly in the form of pairs.

In addition, the determination of the defect concentration 
TABLE I. Some parameters for the five ingots studied: boron and phosphorus concentrations added to the melt, measured resistivities, hole mobilities from Klaassen's model, equilibrium hole concentrations $p_{0}$ determined from the resistivity, and hole mobility, the boron and phosphorus concentrations $N_{A}$ and $N_{D}$ in the wafers determined by GDMS, and $N_{A}$ measured via the iron-acceptor repairing time constants $\tau_{\text {assoc. }}$.

\begin{tabular}{|c|c|c|c|c|c|c|c|c|}
\hline Ingot & $\begin{array}{l}\text { Boron } \\
\text { added to } \\
\text { melt } \\
\left(\mathrm{cm}^{-3}\right)\end{array}$ & $\begin{array}{c}\text { Phosphorus added } \\
\text { to melt } \\
\left(\mathrm{cm}^{-3}\right)\end{array}$ & $\begin{array}{c}\text { Measured } \\
\text { resistivity } \\
\rho \\
(\Omega \mathrm{cm})\end{array}$ & $\begin{array}{c}\mu_{h}\left(\mathrm{~cm}^{2} / \mathrm{V} \mathrm{s}\right) \\
\text { (Klaassen's } \\
\text { model })\end{array}$ & $\begin{array}{c}\text { Equilibrium } \\
\text { hole } \\
\text { conc. } \\
p_{0} \\
\left(\mathrm{~cm}^{-3}\right)\end{array}$ & $\begin{array}{l}N_{A} \text { from } \\
\text { GDMS } \\
\left(\mathrm{cm}^{-3}\right)\end{array}$ & $\begin{array}{l}N_{D} \text { from } \\
\text { GDMS } \\
\left(\mathrm{cm}^{-3}\right)\end{array}$ & $\begin{array}{c}N_{A} \text { from } \\
\tau_{\text {assoc }} \\
\left(\mathrm{cm}^{-3}\right)\end{array}$ \\
\hline 74 & $0.35 \times 10^{16}$ & Nil & $3.5-6.5$ & $435-443$ & $(0.41-0.22) \times 10^{16}$ & $<10^{16}$ & $<10^{15}$ & $0.36 \times 10^{16}$ \\
\hline 72 & $1.75 \times 10^{16}$ & Nil & 1.1 & 409 & $1.4 \times 10^{16}$ & $1.2 \times 10^{16}$ & $<10^{15}$ & $1.7 \times 10^{16}$ \\
\hline 73 & $5.0 \times 10^{16}$ & Nil & 0.44 & 368 & $3.9 \times 10^{16}$ & $4.2 \times 10^{16}$ & $<10^{15}$ & $4.3 \times 10^{16}$ \\
\hline 45 & $3.25 \times 10^{16}$ & $2.75 \times 10^{16}$ & 2.0 & $\cdots$ & $\cdots$ & $3.7 \times 10^{16}$ & $3.3 \times 10^{16}$ & $4.0 \times 10^{16}$ \\
\hline 44 & $7.5 \times 10^{16}$ & $5.0 \times 10^{16}$ & 0.57 & $\cdots$ & $\cdots$ & $7.1 \times 10^{16}$ & $4.5 \times 10^{16}$ & $8.1 \times 10^{16}$ \\
\hline
\end{tabular}

via photoconductance-based carrier lifetime measurements requires knowledge on the carrier mobilities. Measurement of the majority carrier mobilities in compensated silicon, via a combination of free-carrier absorption (FCA) and resistivity measurements, confirmed that the majority carrier mobility is reduced in compensated material, in accordance with the mobility model of Klaassen. ${ }^{24,25}$

\section{EXPERIMENTAL METHODS}

The samples used were cleaved sections of $155 \times$ $155 \mathrm{~mm}^{2}$ pseudosquare, $p$-type, $\langle 100\rangle$-oriented Czochralskigrown silicon $(\mathrm{Cz}-\mathrm{Si})$ wafers. There were wafers from three control ingots (noncompensated), which were boron doped, and also two compensated ingots, doped with both boron and phosphorus. The amounts of dopant added to the melt prior to the growth of these ingots are shown in Table I. Note that the actual dopant concentrations in the wafers will be affected by segregation during ingot growth and will vary from the initial melt concentration. High-purity feedstock and dopant sources were used during ingot growth, hence we do not expect significant quantities of unintended dopants.

The interstitial oxygen concentrations $\left[\mathrm{O}_{i}\right]$ were measured by FTIR and were between $9.4 \times 10^{17}$ and 1.1 $\times 10^{18} \mathrm{~cm}^{-3}$, and hence very similar from wafer to wafer. The measurements were based on the ASTM standard. ${ }^{26}$ In addition to the $\mathrm{Cz}-\mathrm{Si}$ wafers, some boron-doped float-zone wafers (FZ-Si) with boron concentrations of 3.3 $\times 10^{16} \mathrm{~cm}^{-3}$ were also included to act as monitors for any unwanted reduction in surface passivation quality during the experiment, which could otherwise erroneously be attributed to the $\mathrm{B}_{s}-\mathrm{O}_{2 i}$ defect. Such FZ-Si wafers have very low oxygen content and are not subject to the $\mathrm{B}_{s}-\mathrm{O}_{2 i}$ defect.

In preparation for carrier lifetime measurements, several samples from each ingot were etched and cleaned. They were then subject to a phosphorus diffusion step to remove fastdiffusing metal impurities such as interstitial $\mathrm{Fe}\left(\mathrm{Fe}_{i}\right)$, which can otherwise make determination of the $\mathrm{B}_{s}-\mathrm{O}_{2 i}$ defect concentration difficult. This can occur because $\mathrm{Fe}_{i}$ forms pairs with the acceptor atoms at room temperature, which can be broken by the illumination needed to activate the $\mathrm{B}_{s}-\mathrm{O}_{2 i}$ defect. This causes an additional change in the carrier lifetime that is superposed upon that due to the $\mathrm{B}_{s}-\mathrm{O}_{2 i}$ defect. Another benefit of performing gettering is that the electronic quality of the compensated silicon prior to activation of the
$\mathrm{B}_{s}-\mathrm{O}_{2 i}$ defect can be assessed in the absence of additional impurities. The high temperature associated with the phosphorus gettering also eliminates any unwanted thermal donors in the $\mathrm{Cz}-\mathrm{Si}^{7}$.

After etching the gettering layer, all samples were coated at $400{ }^{\circ} \mathrm{C}$ with plasma-enhanced chemical vapor-deposited silicon nitride for surface passivation. The wafers were initially $200 \mu \mathrm{m}$ thick, with a random pyramid texture on both surfaces. After the etching steps described above, the surfaces were planar and the samples had a final thickness of between 130 and $140 \mu \mathrm{m}$. The silicon nitride deposition was followed by a $400{ }^{\circ} \mathrm{C}$ forming gas anneal for $20 \mathrm{~min}$, which we have found improves the surface passivation. Extraction of the samples from the forming gas furnace was performed in the dark to ensure that no activation of the $\mathrm{B}_{s}-\mathrm{O}_{2 i}$ defect occurred due to room light as the samples cooled.

Effective carrier lifetimes were measured with the quasisteady-state photoconductance (QSSPC) technique and the transient photoconductance decay (PCD) technique. ${ }^{27}$ Measurements were performed after extraction from the forming gas anneal furnace in the dark, yielding a measure of the lifetime prior to any $\mathrm{B}_{s}-\mathrm{O}_{2 i}$ defect activation, which we refer to as $\tau_{\text {annealed. }}$. The lifetime was measured again after 2 days of illumination under a halogen lamp with an intensity equivalent to approximately one-tenth of 1 sun. This should be sufficient to fully activate the $\mathrm{B}_{s}-\mathrm{O}_{2 i}$ defect. ${ }^{9}$ This was confirmed via illumination for an extra 3 days, which did not cause any additional reduction in the lifetime. This final lifetime is denoted as $\tau_{\text {degraded }}$.

The relative defect concentration $N_{t}^{*}$ can then be determined via $N_{t}^{*}=1 / \tau_{\text {degraded }}-1 / \tau_{\text {annealed }}$, where by convention ${ }^{7}$ the carrier lifetimes are measured at an excess carrier density equal to $10 \%$ of the net doping $p_{0}$ (note that it is the net doping rather than the total acceptor concentration that determines the recombination activity of a given defect).

Typical resistivities of the wafers used in this work, as measured via the dark conductance, are shown in Table I. A range is shown for the higher resistivity controls since they were somewhat variable from wafer to wafer and depended slightly on the thermal history (due to thermal donor annihilation). The resistivities of wafers from the other ingots were more uniform and stable. Assuming that the majority carrier (hole) mobilities in the noncompensated control samples are equal to the values expected from Klaassen's mobility 
model, ${ }^{24,25}$ the corresponding boron concentrations $N_{A}$ can be determined for the control samples, and are also shown in Table I. We have chosen to use Klaassen's mobility model in this work, as it accounts for the different scattering cross sections of minority and majority dopant atoms and so should in principle be applicable to compensated silicon.

Table I also shows independent measurements of the boron and phosphorus concentrations $N_{A}$ and $N_{D}$ in similar wafers from both the control and compensated ingots by glowdischarge mass spectrometry (GDMS) performed at SINTEF. ${ }^{28,29}$ The samples were presputtered for $5 \mathrm{~min}$ to remove surface contamination, with a sputtering rate of approximately $20 \mathrm{~nm} / \mathrm{s}$. The relative sensitivity factors for this apparatus applied to a silicon matrix have recently been found to be 1.5 for boron and 1.1 for phosphorus.

$N_{A}$ was also determined by the recently proposed technique based on measuring the iron-acceptor pair association time constant $\tau_{\text {assoc }}{ }^{30}$ This technique in principle allows for the measurement of the total acceptor concentration even in the presence of compensation, since the additional B atoms remain ionized and hence Coulombically attractive to positively charged $\mathrm{Fe}_{i}$. The surprising fact that the technique appears to work even in the suggested presence of B-P complexes is discussed below.

Note that the samples used for the GDMS and Feacceptor pairing were of slightly different resistivities to those studied here, although they were from the same ingots. Nevertheless, the $N_{A}$ values agree reasonably well with those determined from the resistivity for the control wafers. The $N_{A}$ values determined by these techniques for the compensated samples will prove useful in the analysis below. All lifetime and resistivity measurements were performed at $304 \pm 2 \mathrm{~K}$, and the mobilities calculated with Klaassen's mobility model $^{24,25}$ were determined at this temperature also.

A key parameter for the analysis below is the net doping $p_{0}$. It could, in principle, be estimated by subtracting the boron and phosphorus concentrations determined by GDMS, but this is rather inaccurate, especially when the compensation is strong. Resistivity measurements alone are not sufficient since the majority carrier mobility is affected by compensation to a degree that is not well known. In this paper we have used FCA measured by a Cary 5 spectrophotometer fitted with an integrating sphere to estimate $p_{0}$ in the compensated samples by direct comparison to the FCA observed in the noncompensated controls and by assuming a value for $p_{0}$ for the control wafers based on the resistivity data and hole mobilities from Klaassen's model, shown in Table I. Our wafers are thin, and the FCA is not strong in the near-IR region. Hence we used the original $200 \mu \mathrm{m}$ thick textured wafers with a mirror placed behind them to significantly increase the optical path length of the infrared photons and increase the absorptance to a sufficiently noise-free level.

\section{RESULTS AND DISCUSSION}

A difficulty in applying the photoconductance-based lifetime measurement techniques used here (whether QSSPC or PCD) to compensated samples is that these techniques require a knowledge on the majority and minority carrier mo-

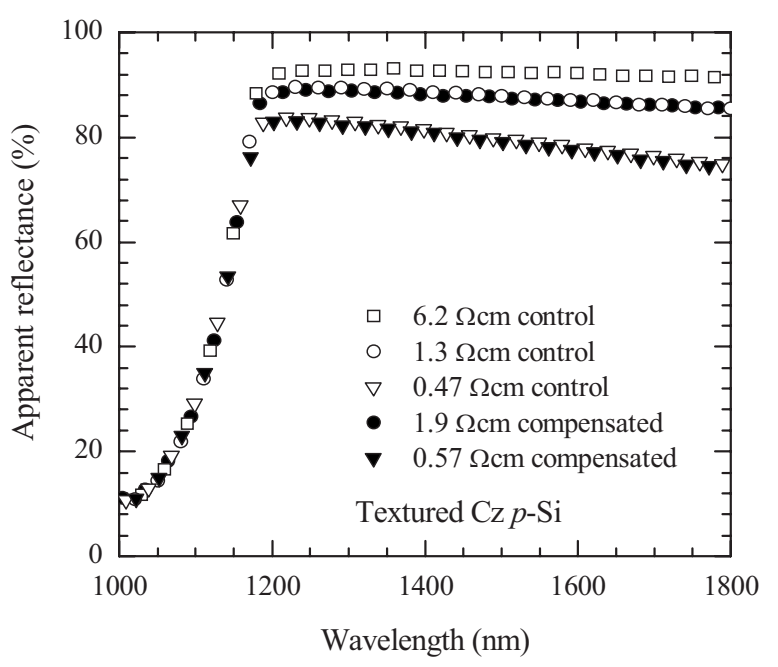

FIG. 1. Apparent reflectance as a function of wavelength measured on textured wafers with a mirror placed behind them.

bility sum $\mu_{h}+\mu_{e}$. This is necessary in order to determine the excess carrier density $\Delta n$ from the conductance. The values of $\Delta n$ must be known quite accurately to enable the carrier lifetime to be extracted at an excess carrier density equal to $10 \%$ of the net doping $p_{0}$ in the sample. In fact, in the case of the QSSPC technique, the lifetime itself, as well as the excess carrier density, depends on the mobility sum. Many of our samples had effective lifetimes of less than $100 \mu \mathrm{s}$, meaning we could only apply the QSSPC approach (since the decay of the transient flash is too slow to allow accurate transient measurements in such cases). For noncompensated crystalline silicon, the default mobility model routinely used in the analysis of photoconductance lifetimes is a parametrization based on the measured data of Dannhauser and Krausse. ${ }^{31,32}$ However, this mobility model is not strictly valid for compensated silicon since it does not account for the different scattering cross sections of majority and minority dopant atoms. Hence, we have developed a procedure for estimating the mobility sum in the compensated material in order to generate more accurate lifetime and $\Delta n$ values from the measured photoconductance.

The following sections describe our measurements of $p_{0}$ via FCA and then the use of this information, coupled with the resistivity, to determine the majority carrier mobilities in the compensated wafers. These values were then compared with those from Klaassen's mobility model, ${ }^{24,25}$ which in principle can account for scattering from additional compensated majority and minority dopant atoms. A reasonable agreement then prompted us to use this same mobility model to estimate the minority carrier mobilities, yielding the mobility sum, and allowing us to determine the lifetimes at the required excess carrier densities.

\section{A. Measuring the net doping $p_{0}$}

Figure 1 plots the "apparent" reflectance $R$ as a function of wavelength measured on textured samples from each ingot, with a mirror positioned behind the samples. The small amount of light absorbed by the mirror has been corrected for. Note that the apparent reflectance measurement does not 


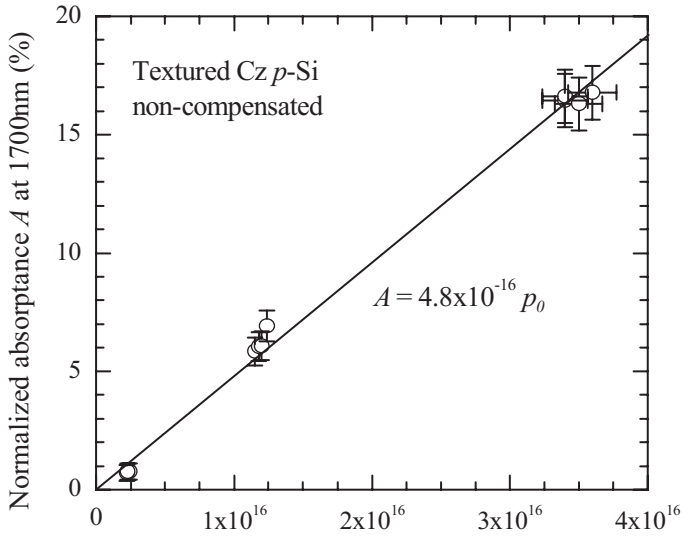

Equilibrium majority carrier concentration $p_{0}=N_{A}\left(\mathrm{~cm}^{-3}\right)$

FIG. 2. Normalized absorptance at $1700 \mathrm{~nm}$ as a function of the equilibrium hole concentration $p_{0}$ for the noncompensated control samples. The solid line is a linear fit to the data.

only include primary reflections from the front surface but also trapped photons which pass multiple times within the sample and which eventually re-emerge through the front surface. Below $1200 \mathrm{~nm}$ the reflectance decreases rapidly due to electron-hole pair generation within the silicon, while above $1200 \mathrm{~nm}$, the reflectance is dominated by FCA. In this region, the samples with a greater net doping concentration show a reduced reflectance.

Since there can be almost no transmission of light through the mirror, the absorptance is $A=1-R$. Figure 2 shows the "normalized" absorptance at $1700 \mathrm{~nm}$ for the noncompensated samples only as a function of $p_{0}$. The normalization firstly involved a linear correction for small variations in the sample thickness. We also subtracted a fixed fraction of the incident light which escapes through the sides of the sample (not a negligible fraction due to the excellent light-trapping produced by the surface texture). This "leakage" was found to be approximately $8 \%$. In general, the fraction of leaked light will also depend weakly on the amount of FCA, and hence the net doping, but we have neglected this small correction here.

The result is an approximately linear relationship between $p_{0}$ and the normalized absorptance, as shown by the straight line in Fig. 2. By measuring the absorptance in the compensated samples, we may use this linear fit to estimate the $p_{0}$ for those samples also. The results are shown in Table II. Note the strong compensation in these wafers, for ingot 45 , approximately $75 \%$ of the boron is compensated by phosphorus, whereas for ingot 44 , about $50 \%$ of the boron is compensated. Based on estimates for the uncertainty and random noise in the reflectance measurements, and the uncertainty in the $p_{0}$ values for the noncompensated wafers (5\%), the uncertainty in $p_{0}$ for the compensated samples is approximately $10 \%$. Note that a linear relationship between $p_{0}$ and the absorption coefficient has been confirmed previously for $p$-type silicon with hole concentrations in the range observed here. $^{33}$

\section{B. Determining the carrier mobilities and lifetimes}

We may then proceed to determine the majority carrier (hole) mobility for the compensated samples, via the resistivity $\rho$ and $p_{0}$ measurements, using $1 / \rho=q \dot{\mu}_{h} p_{0}$, with $q$ as the elementary charge. The results are $\mu_{h}=319 \pm 40$ and $303 \pm 30 \mathrm{~cm}^{2} / \mathrm{V} \mathrm{s}$ for ingots 45 and 44 , respectively, as listed in Table II. These may be compared to the predicted hole mobilities from Klaassen's model, using as input parameters the $N_{A}$ values estimated from the iron-acceptor pair association time constant $\tau_{\text {assoc }}$, and $N_{D}$ values determined via $N_{D}=N_{A}-p_{0}$, as also listed in Table II (note that these $N_{A}$ and $N_{D}$ values are in good agreement with the GDMS values in Table I). The results are $\mu_{h}=347$ and $307 \mathrm{~cm}^{2} / \mathrm{V} \mathrm{s}$ for ingots 45 and 44, respectively, in very good agreement with our measured mobility values. By way of comparison, in the absence of compensation, Klaassen's model predicts that $p$-type samples with the same values of $p_{0}$ would have hole mobilities of 419 and $372 \mathrm{~cm}^{2} / \mathrm{V} \mathrm{s}$ for ingots 45 and 44 , respectively, revealing that the compensation does have a significant impact on mobility. Our measured mobilities are $24 \%$ and $19 \%$ lower than these values. Lower mobilities have been reported in compensated silicon previously. ${ }^{34-36}$

The results above imply that Klaassen's model yields reasonable estimates of the majority carrier mobilities in the compensated samples. We then propose to trust it further by using it to estimate the electron mobility, thus yielding the mobility sum $\mu_{h}+\mu_{e}$ that is needed in calculating the carrier lifetime from the photoconductance data. ${ }^{27}$ The mobility sums are also shown in Table II.

We are then able to calculate the carrier lifetimes from measured photoconductance data for both the noncompensated and compensated samples. These are shown in Fig. 3, with all lifetimes reported at an excess carrier density equal to $10 \%$ of the net doping $p_{0}$. Lifetimes are reported both before and after activation of the $\mathrm{B}_{s}-\mathrm{O}_{2 i}$ defect. Firstly note that the FZ control samples are not degraded, as expected

TABLE II. Measured and estimated parameters for the two compensated ingots: measured resistivities, net doping from FCA data, the corresponding hole mobilities $\mu_{h}, N_{A}$ measured via the iron-acceptor repairing time constants $\tau_{\text {assoc }} N_{D}$ determined via $N_{A}-p_{0}$ hole mobilities $\mu_{h}$ from Klaassen's model with $N_{A}$ and $N_{D}$ as input parameters, and the mobility sum $\mu_{h}+\mu_{e}$ from Klaassen's model.

\begin{tabular}{cccccccc}
\hline \hline Measured & $\begin{array}{c}\text { Net doping } \\
p_{0} \text { from } \\
\text { Ingot }\end{array}$ & $\begin{array}{c}\text { Measured } \\
(\Omega \mathrm{cm})\end{array}$ & $\begin{array}{c}N_{A} \text { from } \\
\left(\mathrm{cm}^{-3}\right)\end{array}$ & $\begin{array}{c}\tau_{\text {assoc }} \\
\mu_{h}\left(\mathrm{~cm}^{2} / \mathrm{V} \mathrm{s}\right)\end{array}$ & $\begin{array}{c}N_{D}=N_{A}-p_{0} \\
\left(\mathrm{~cm}^{-3}\right)\end{array}$ & $\begin{array}{c}\mu_{h}\left(\mathrm{~cm}^{2} / \mathrm{V} \mathrm{s}\right) \\
(\mathrm{Klaassen}) \\
\text { model })\end{array}$ & $\begin{array}{c}\mu_{h}+\mu_{e} \\
\left(\mathrm{~cm}^{2} / \mathrm{V} \mathrm{s}\right) \\
(\text { Klaassen })\end{array}$ \\
\hline 45 & 2.0 & $0.98 \times 10^{16}$ & 319 & $4.0 \times 10^{16}$ & $3.0 \times 10^{16}$ & 347 & 1169 \\
44 & 0.57 & $3.6 \times 10^{16}$ & 303 & $8.1 \times 10^{16}$ & $4.5 \times 10^{16}$ & 307 & 980 \\
\hline \hline
\end{tabular}




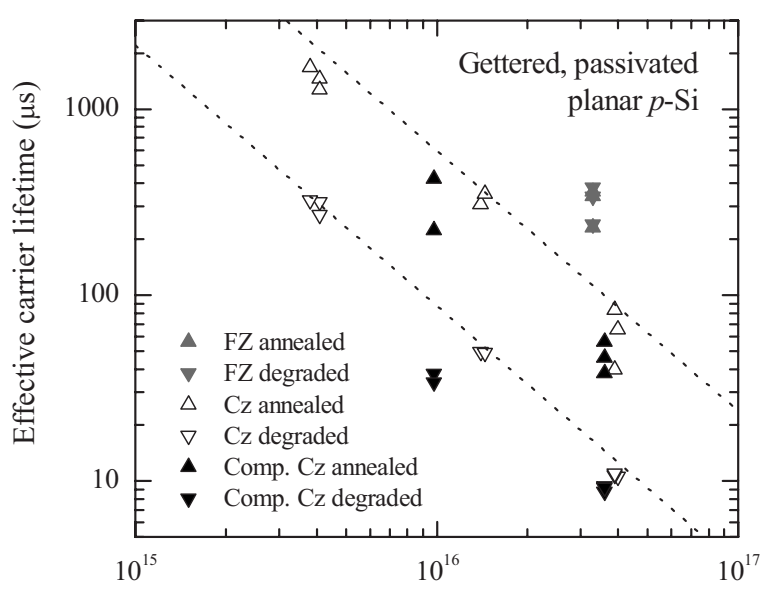

Equilibrium majority carrier concentration $p_{0}=N_{A}-N_{D}\left(\mathrm{~cm}^{-3}\right)$

FIG. 3. Effective carrier lifetimes measured at an excess carrier density of $\Delta n=0.1 \times p_{0}$, as a function of $p_{0}$ for the noncompensated and compensated samples, and also the FZ controls. Lifetimes in the annealed state and after light-induced degradation are shown. The dashed lines are guides to the eye indicating the approximate upper limit on the lifetimes in the noncompensated $\mathrm{Cz}$ wafers.

due to their low oxygen content. This allows us to attribute any reduction in lifetimes in the $\mathrm{Cz}$ samples to the $\mathrm{B}_{s}-\mathrm{O}_{2 i}$ defect rather than, for example, instabilities in the $\mathrm{SiN}$ surface passivation.

After degradation, the lifetimes are reduced by approximately an order of magnitude in both the compensated and noncompensated $\mathrm{Cz}$ wafers. These lifetimes are subsequently used to determine the $\mathrm{B}_{s}-\mathrm{O}_{2 i}$ defect concentration $N_{t}^{*}$. However, at this point it is interesting to note that the lifetimes in the compensated samples are lower than, but not far below, those observed in the control samples, as indicated by the dashed lines in Fig. 3. This implies that even quite strongly compensated material could, in principle, be suitable for solar cell fabrication, notwithstanding reductions in the carrier mobilities.

\section{Determining the defect concentration $\boldsymbol{N}_{t}^{*}$}

It is now straightforward to determine the $\mathrm{B}_{s}-\mathrm{O}_{2 i}$ defect concentration $N_{t}^{*}$ via the expression $N_{t}^{*}=1 / \tau_{\text {degraded }}-$ $1 / \tau_{\text {annealed }}$. The results are shown in Fig. 4. For the noncompensated control samples, the defect concentration increases approximately linearly with the boron concentration $N_{A}$, as expected. ${ }^{8}$ The dashed line is fitted through the control data to provide a comparison for the compensated data. The $N_{t}^{*}$ values for the compensated samples are shown plotted as a function of the net doping $p_{0}$ and also plotted against the boron concentration $N_{A}$ as determined from the iron-acceptor association time $\tau_{\text {assoc }}$.

If the $\mathrm{B}_{s}-\mathrm{O}_{2 i}$ defects were able to form with all of the boron in the samples, then the compensated data plotted as a function of $N_{A}$ should line up with the control data. However, this is clearly not the case. In fact, the compensated data are in reasonable agreement with the control data when plotted against $p_{0}$. This implies that it is only the uncompensated boron that is available to form the $\mathrm{B}_{s}-\mathrm{O}_{2 i}$ defect or, conversely, that the compensated boron must be present in a

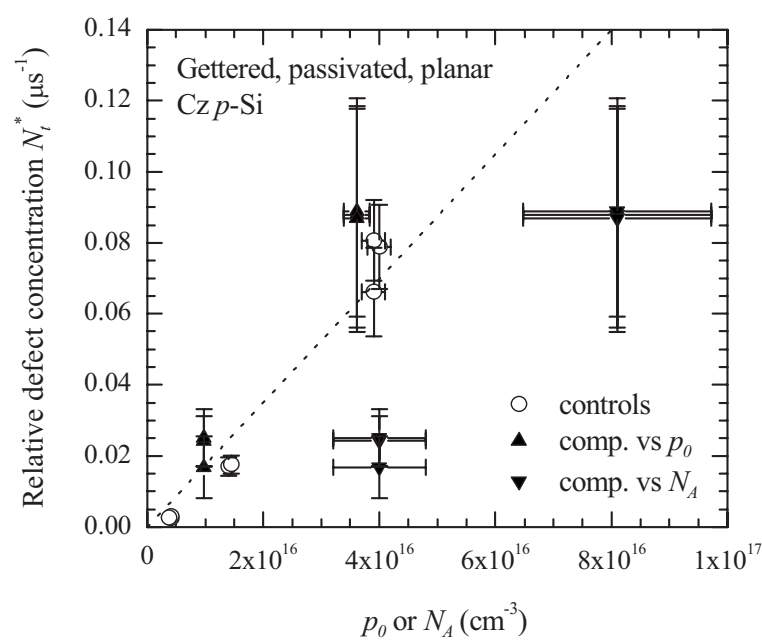

FIG. 4. Relative defect concentration $N_{t}^{*}$ as a function of either $p_{0}$ or $N_{A}$ for both the noncompensated controls and the compensated samples. The dashed line is a linear fit to the control data.

form that cannot bond with oxygen dimers (or at least if it does, it forms a complex that is not recombination active). This observation could be explained by the presence of B-P complexes.

The uncertainties in $N_{t}^{*}$ shown in Fig. 4 were calculated by assuming an uncertainty in the carrier lifetime measurements of $10 \%$ for the control samples, which is a conservative estimate for QSSPC measurements. ${ }^{37}$ An uncertainty of $20 \%$ in the lifetime measurements was assumed for the compensated samples due to additional uncertainty in the mobility sum. Uncertainties in the $p_{0}$ values for the control samples were $5 \%$ and $10 \%$ for the compensated samples, as described above. The uncertainty in $N_{A}$ for the compensated samples derived from iron-acceptor repairing was estimated at $20 \% .^{30}$

\section{The defect generation rate}

Further indirect evidence for B-P complexes comes from measurements of the generation rate of the $\mathrm{B}_{s}-\mathrm{O}_{2 i}$ defect. An attractive aspect of this approach is that it does not require knowledge on the absolute value of the lifetime or injection level, but only a measurement of the relative change in lifetime, from which a time constant is extracted. Hence modifications to the carrier mobilities are not required for accurate results, in contrast to the analysis above regarding the defect concentrations.

The generation rate of the $\mathrm{B}_{s}-\mathrm{O}_{2 i}$ defect has been shown to be proportional to $p_{0} \times N_{A}{ }^{38}$ Here, $N_{A}$ is implicitly understood to mean the concentration of boron atoms available for defect generation. The dependence on $p_{0}$ comes from the requirement for the oxygen dimers to capture free holes to facilitate their diffusion. An assumption in the model is that the oxygen dimer concentration is much less than $p_{0}$, which will certainly be true for the samples studied here. In compensated $p$-type silicon, if all of the phosphorus atoms are complexed with boron atoms, then the amount of boron available for defect formation is simply $p_{0}$, and the generation rate will be proportional to $p_{0}^{2}$. On the other hand, if all of the boron is isolated, and hence able to bond with the 


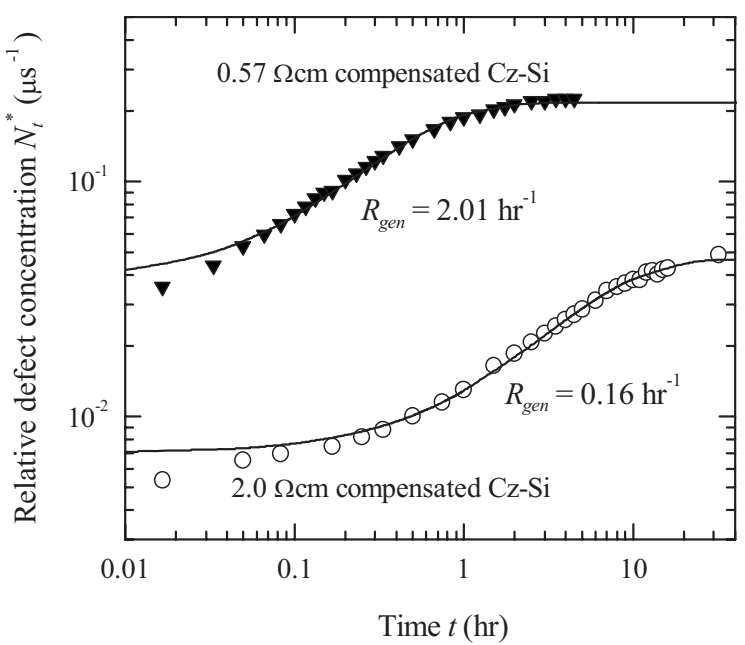

FIG. 5. Relative defect concentration $N_{t}^{*}$ as a function of time for two compensated samples, as the defect forms under illumination. The solid lines show fits used to determine the defect generation rates.

oxygen dimers, then the generation rate will be proportional to $p_{0} \times N_{A}$.

The generation rate of the defect $R_{\text {gen }}$ is determined by fitting a curve of the form $N_{t}^{*}(t)=A+B\left[1-\exp \left(-R_{\text {gen }} t\right)\right]$ to measurements of the increase in defect concentration under illumination, where $A$ and $B$ are constants. For these measurements, the white-light illumination intensity was approximately $10 \mathrm{~mW} \mathrm{~cm}{ }^{-2}$, and the temperature was $25^{\circ} \mathrm{C}$. Examples are shown in Fig. 5 for wafers from the two compensated ingots. Note that our data could be fitted quite well with such a single exponential, unlike the light-induced degradation observed recently in compensated solar cells made with solar-grade silicon, ${ }^{39}$ which also contains additional impurities other than extra dopants.

Figure 6 shows defect generation rate values determined for the noncompensated controls and also for the two compensated ingots, plotted either against $p_{0}^{2}$, or against $p_{0} N_{A}$. Figure 6 also shows $R_{\text {gen }}$ data for noncompensated material taken from Bothe et al. ${ }^{9}$ and Rein et al. ${ }^{40}$ The solid line

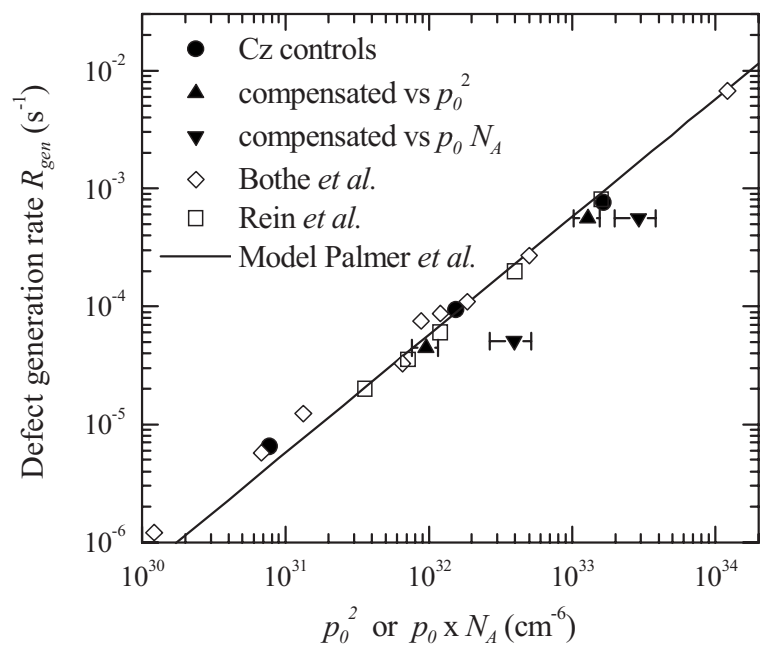

FIG. 6. The defect generation rate $R_{\text {gen }}$, as a function of $p_{0}^{2}$ or $p_{0} N_{A}$. Data for noncompensated wafers taken from Bothe et al. (Ref. 9) and Rein et al. (Ref. 40) are also shown, as is the model of Palmer et al. (Ref. 38). represents the model of Palmer et al. ${ }^{38}$ All of the data for noncompensated silicon follows this model quite well. However, for the case of the compensated material, when plotted against $p_{0} N_{A}$, there is a clear discrepancy. When plotted against $p_{0}^{2}$, the data match the expected model. This reveals that the excess boron is not able to form the $\mathrm{B}_{s}-\mathrm{O}_{2 i}$ defect in these samples. In other words, it excludes the possibility that the dimers still bond with the B-P complexes, and that this complex is not active in terms of recombination. This possibility could not be excluded on the basis of the defect concentration measurements above.

\section{E. Discussion}

The proposed presence of B-P complexes raises one immediate question-why do these complexes apparently prevent the formation of the $\mathrm{B}_{s}-\mathrm{O}_{2 i}$ defect, while the formation of $\mathrm{Fe}_{i}$-acceptor pairs proceeds unaffected? In other words, why does the $\mathrm{B}_{s}-\mathrm{O}_{2 i}$ defect formation rate depend on $p_{0}$, while the $\mathrm{Fe}_{i}$-acceptor pair formation rate depends on $N_{A}$ ? A possible explanation is that both the $\mathrm{B}$ and $\mathrm{P}$ atoms in the B-P complexes remain ionized, forming a type of "dipole" in the silicon lattice. Then the B atoms would remain negatively charged, acting as a coulombic attractor for $\mathrm{Fe}_{i}$, just as isolated boron atoms do. Since the $\mathrm{Fe}_{i}$-acceptor repairing rate is independent of the "acceptor" species, ${ }^{41}$ the measured repairing time will not be affected if some of the boron is in the form of B-P complexes even if the recombination activity of the resulting "Fe-B-P" complexes may be different from the normal FeB pairs.

Why then is the oxygen dimer not able to bind with these $\mathrm{B}-\mathrm{P}$ complexes? The $\mathrm{B}$ atom remains charged, which is necessary for the formation of the defect. ${ }^{38}$ However, it may be that there is insufficient space around the B-P complex for the dimer to bond, or that the presence of the negatively charged $\mathrm{P}$ atom "behind" the $\mathrm{B}$ atom does not allow the dimer to properly "wrap around" the B atom. Interestingly, the lack of space around larger acceptor species is believed to be the reason why there is no $\mathrm{Ga}_{s}-\mathrm{O}_{2 i}$ analog to the $\mathrm{B}_{s}-\mathrm{O}_{2 i}$ defect in Ga-doped $p$-type silicon. ${ }^{5}$ On the other hand, Fe-Ga pairs are known to form readily in such material. ${ }^{41}$ Detailed computer modeling, for example, using local electron-density functional theory, ${ }^{42}$ would be required to verify this possibility.

Finally, a further implication of the possible presence of B-P complexes is that there may be fewer scattering centers to reduce the carrier mobilities, compared to the case where the $\mathrm{B}$ and $\mathrm{P}$ remain isolated.

\section{CONCLUSION}

The concentration of $\mathrm{B}_{s}-\mathrm{O}_{2 i}$ defects that are created in compensated $p$-type silicon under illumination is approximately proportional to the net doping $p_{0}$ rather than the total boron concentration $N_{A}$. In addition, the rate at which these defects form is proportional to $p_{0}^{2}$, rather than $p_{0} \times N_{A}$, the latter being expected if all of the boron is able to form complexes with the oxygen dimers. These observations, combined, strongly imply that the compensated boron is unable to form the $\mathrm{B}_{s}-\mathrm{O}_{2 i}$ defect. This could be explained by the 
presence of B-P complexes in the material, which, if they exist, must form at very high temperatures during ingot growth and cooling. Although such complexes apparently prohibit the formation of $\mathrm{B}_{s}-\mathrm{O}_{2 i}$ defects, they still evidently bond with interstitial iron, as shown previously. ${ }^{30}$

Whatever the cause of the lower than expected activity of $\mathrm{B}_{s}-\mathrm{O}_{2 i}$ defects in compensated $p$-type silicon, the implications for solar cells are positive. However, an additional problem with using compensated material is the reduced mobilities. We have measured small reductions in the majority carrier mobility here (about 20\%). For devices, however, the minority carrier mobility is often more important, since it determines the minority carrier diffusion length. A similar $20 \%$ reduction in the minority carrier mobility would result in a modest $10 \%$ reduction in the minority carrier diffusion length. However, the impact of compensation on minority carrier mobilities remains to be confirmed.

\section{ACKNOWLEDGMENTS}

D.M. is supported by an Australian Research Council QEII Fellowship, L.J.G. acknowledges SenterNovem for support, and B.L. and J.S. acknowledge the support of the German Academic Exchange Service. The authors are grateful to Kai Petter of Q-cells for kindly providing the silicon wafers, Chris Samundsett of ANU for sample preparation, and Ron Sinton of Sinton Consulting for providing a QSSPC data analysis package with easily adjusted mobilities.

${ }^{1} \mathrm{H}$. Fischer and W. Pschunder, Proceedings of the Tenth IEEE Photovoltaic Specialists Conference, Palo Alto, CA (IEEE, New York, 1973), p. 404.

${ }^{2}$ J. Schmidt, C. Berge, and A. G. Aberle, Appl. Phys. Lett. 73, 2167 (1998).

${ }^{3}$ S. Rein and S. W. Glunz, Appl. Phys. Lett. 82, 1054 (2003).

${ }^{4} \mathrm{~J}$. Schmidt, K. Bothe, and R. Hezel, Proceedings of the 29th IEEE Photovoltaic Specialists Conference, New Orleans, LA (IEEE, New York, 2002), p. 178 .

${ }^{5}$ J. Schmidt and K. Bothe, Phys. Rev. B 69, 024107 (2004).

${ }^{6}$ D. Macdonald, L. J. Geerligs, and S. Reipe, Proceedings of the 13th Workshop on Crystalline Silicon Solar Cell Materials and Processes, Vail, CO (NREL, Golden, CO, 2003), p. 182.

${ }^{7}$ K. Bothe, R. Sinton, and J. Schmidt, Prog. Photovoltaics 13, 287 (2005).

${ }^{8}$ K. Bothe and J. Schmidt, Appl. Phys. Lett. 87, 262108 (2005).

${ }^{9}$ K. Bothe and J. Schmidt, J. Appl. Phys. 99, 013701 (2006).

${ }^{10}$ A. Herguth, G. Schubert, M. Kaes, and G. Hahn, Prog. Photovoltaics 16, 135 (2008)

${ }^{11}$ N. Yuge, M. Abe, K. Hanazawa, H. Baba, N. Nakamura, Y. Kato, Y. Sakaguchi, S. Hiwasa, and F. Aratani, Prog. Photovoltaics 9, 203 (2001).

${ }^{12}$ D. Sarti and R. Einhaus, Sol. Energy Mater. Sol. Cells 72, 27 (2002).

${ }^{13}$ S. Pizzini, M. Acciarri, and S. Binetti, Phys. Status Solidi A 202, 2928 (2005).

${ }^{14}$ J. Degoulange, I. Perichaud, C. Trassy, and S. Martinuzzi, Sol. Energy Mater. Sol. Cells 92, 1269 (2008).
${ }^{15}$ S. Dubois, N. Enjalbert, and J. P. Garandet, Appl. Phys. Lett. 93, 032114 (2008).

${ }^{16}$ R. Kopecek, J. Arumughan, K. Peter, E. A. Good, J. Libal, M. Acciarri, and S. Binetti, Proceedings 23rd European Photovoltaic Solar Energy Conference, Valencia, Spain (WIP-Renewable Energies, Munich, Germany, 2008), p. 1855.

${ }^{17}$ S. Dubois, N. Enjalbert, J. P. Garandet, R. Monna, and J. Kraiem, Proceedings of the 23rd European Photovoltaic Solar Energy Conference, Valencia, Spain (WIP-Renewable Energies, Munich, Germany, 2008), p. 1437.

${ }^{18}$ E. A. Good, R. Kopecek, and J. Arumughan, Proceedings of the 23rd European Photovoltaic Solar Energy Conference, Valencia, Spain (WIPRenewable Energies, Munich, Germany, 2008), p. 1218.

${ }^{19}$ W. Krühler, C. Moser, F. W. Schulze, and H. Aulich, in Proceedings of the Eighth European Photovoltaic Solar Energy Conference, Florence, Italy (Kluwer Academic, Dordrecht, The Netherlands, 1988), p. 1181.

${ }^{20}$ R. C. Newman and R. S. Smith, Solid State Commun. 5, 723 (1967).

${ }^{21}$ V. Tsvetov, W. Allred, and W. G. Spitzer, Appl. Phys. Lett. 10, 326 (1967).

${ }^{22}$ J. F. Morhange, R. Beserman, and M. Balkanski, Phys. Status Solidi A 23, 383 (1974)

${ }^{23}$ M. Jouanne, J. F. Morhange, and M. Balkanski, Phys. Status Solidi B 92, 255 (1979)

${ }^{24}$ D. B. M. Klaassen, Solid-State Electron. 35, 953 (1992).

${ }^{25}$ D. B. M. Klaassen, Solid-State Electron. 35, 961 (1992).

${ }^{26}$ ASTM Standard 1188-93a, Standard Test Method for Interstitial Atomic Oxygen Content of Silicon by Infrared Absorption, ASTM International, West Conshohocken, PA, 2003 (1993).

${ }^{27}$ R. A. Sinton and A. Cuevas, Appl. Phys. Lett. 69, 2510 (1996).

${ }^{28}$ M. Di Sabatino, A. L. Dons, J. Hinrichs, O. Lohne, and L. Arnberg, Proceedings of the 22nd European Photovoltaic Solar Energy Conference, Milan, Italy (WIP-Renewable Energies, Munich, Germany, 2007), p. 271.

${ }^{29}$ D. Macdonald, A. Cuevas, M. Di Sabatino, and L. J. Geerligs, Proceedings of the 23rd European Photovoltaic Solar Energy Conference, Valencia, Spain (WIP-Renewable Energies, Munich, Germany, 2008), p. 951.

${ }^{30}$ D. Macdonald, A. Cuevas, and L. J. Geerligs, Appl. Phys. Lett. 92, 202119 (2008).

${ }^{31}$ F. Dannhauser, Solid-State Electron. 15, 1371 (1972).

${ }^{32}$ J. Krausse, Solid-State Electron. 15, 1377 (1972).

${ }^{33}$ H. B. Briggs, Phys. Rev. 77, 727 (1950).

${ }^{34}$ S. Pizzini and C. Calligarich, J. Electrochem. Soc. 131, 2128 (1984).

${ }^{35}$ J. Libal, S. Novaglia, M. Acciarri, S. Binetti, R. Petres, J. Arumughan, R. Kopecek, and A. Prokopenko, J. Appl. Phys. 104, 104507 (2008).

${ }^{36}$ K. Peter, R. Kopecek, A. Soiland, and E. Enebakk, Proceedings of the 23rd European Photovoltaic Solar Energy Conference, Valencia, Spain (WIP-Renewable Energies, Munich, Germany, 2008), p. 947.

${ }^{37}$ K. R. McIntosh and R. A. Sinton, in Proceedings of the 23rd European Photovoltaic Solar Energy Conference, Valencia, Spain (WIP-Renewable Energies, Munich, Germany, 2008), p. 77.

${ }^{38}$ D. W. Palmer, K. Bothe, and J. Schmidt, Phys. Rev. B 76, 035210 (2007).

${ }^{39}$ S. Dubois, N. Enjalbert, and J. P. Garandet, Appl. Phys. Lett. 93, 103510 (2008).

${ }^{40}$ S. Rein, T. Rehrl, W. Warta, S. W. Glunz, and G. Willeke, Proceedings of the 17th European Photovoltaic Solar Energy Conference, Munich, Germany (WIP, Munich, 2001), p. 1555.

${ }^{41}$ D. Macdonald, T. Roth, P. Deenapanray, K. Bothe, P. Pohl, and J. Schmidt, J. Appl. Phys. 98, 083509 (2005).

${ }^{42}$ J. Adey, R. Jones, D. W. Palmer, P. R. Briddon, and S. öberg, Phys. Rev. Lett. 93, 055504 (2004). 